

\section{DISCLAIMER}

This report was prepared as an account of work sponsored by an agency of the United States Government. Neither the United States Government nor any agency Thereof, nor any of their employees, makes any warranty, express or implied, or assumes any legal liability or responsibility for the accuracy, completeness, or usefulness of any information, apparatus, product, or process disclosed, or represents that its use would not infringe privately owned rights. Reference herein to any specific commercial product, process, or service by trade name, trademark, manufacturer, or otherwise does not necessarily constitute or imply its endorsement, recommendation, or favoring by the United States Government or any agency thereof. The views and opinions of authors expressed herein do not necessarily state or reflect those of the United States Government or any agency thereof. 


\section{DISCLAIMER}

Portions of this document may be illegible in electronic image products. Images are produced from the best available original document. 


\section{DISCLAIMER}

This report was prepared as an account of work sponsored by an agency of the United States Government. Neither the United States Government nor any agency thereof, nor any of their employees, makes any warranty, express or implied, or assumes any legal liability or responsibility for the accuracy, completeness, or usefulness of any information, apparatus, product, or process disclosed, or represents that its use would not infringe privately owned rights. Reference herein to any specific commercial product, process, or service by trade name, trademark, manufacturer, or otherwise, does not necessarily constitute or inply its endorsement, recommendation, or favoring by the United States Government ar any agency thereof. The views and opinions of authors expressed herein do not necessarily state or reflect those of the United States Government or any agency thereof.

Printed in the United States of America

Available from:

National Technical Information Service

U.S. Department of Commerce 5285 Port Royal Road Springfield, VA 22161

DOMESTIC PRICE CODES

Standard Priced Documents (Schedule A)

Effective January 1, 1982

Prices are for customers in the United States, Canada, and Mexico.

\begin{tabular}{lr} 
Code & Price \\
\hline A01 & $\$ 4.00$ \\
A02 & 6.00 \\
A03 & 7.50 \\
A04 & 9.00 \\
A05 & 10.50 \\
A06 & 12.00 \\
A07 & 13.50 \\
A08 & 15.00 \\
A09 & 16.50 \\
A10 & 18.00 \\
A11 & 19.50 \\
Al2 & 21.00
\end{tabular}

Page Range
Microfiche
$001-025$
$026-050$
$051-075$
$076-100$
$101-125$
$126-150$
$151-175$
$176-200$
$201-225$
$226-250$
$251-275$

\begin{tabular}{lr} 
Code & Price \\
\hline A13 & $\$ 22.50$ \\
A14 & 24.00 \\
A15 & 25.50 \\
A16 & 27.00 \\
A17 & 28.50 \\
A18 & 30.00 \\
A19 & 31.50 \\
A20 & 33.00 \\
A21 & 34.50 \\
A22 & 36.00 \\
A23 & 37.50 \\
A24 & 39.00 \\
A25 & 40.50
\end{tabular}

Page Range

$276-300$

$301-325$

$326-350$

$351-375$

$376-400$

$401-425$

$426-450$

$451-475$

$476-500$

$501-525$

$526-550$

$551-575$

$576-600$

For documents over 600 pages, add $\$ 1.50$ for each additional 25-page increment. 
$\mathrm{PFC} / \mathrm{CP}-82-2$

DOE/ET/51013-38

UC20 G

ANTENNA-PLASMA COUPLING THEORY FOR ICRF HEATING

OF LARGE TOKAMAKS*

A. Ram and A. Bers

March 1982

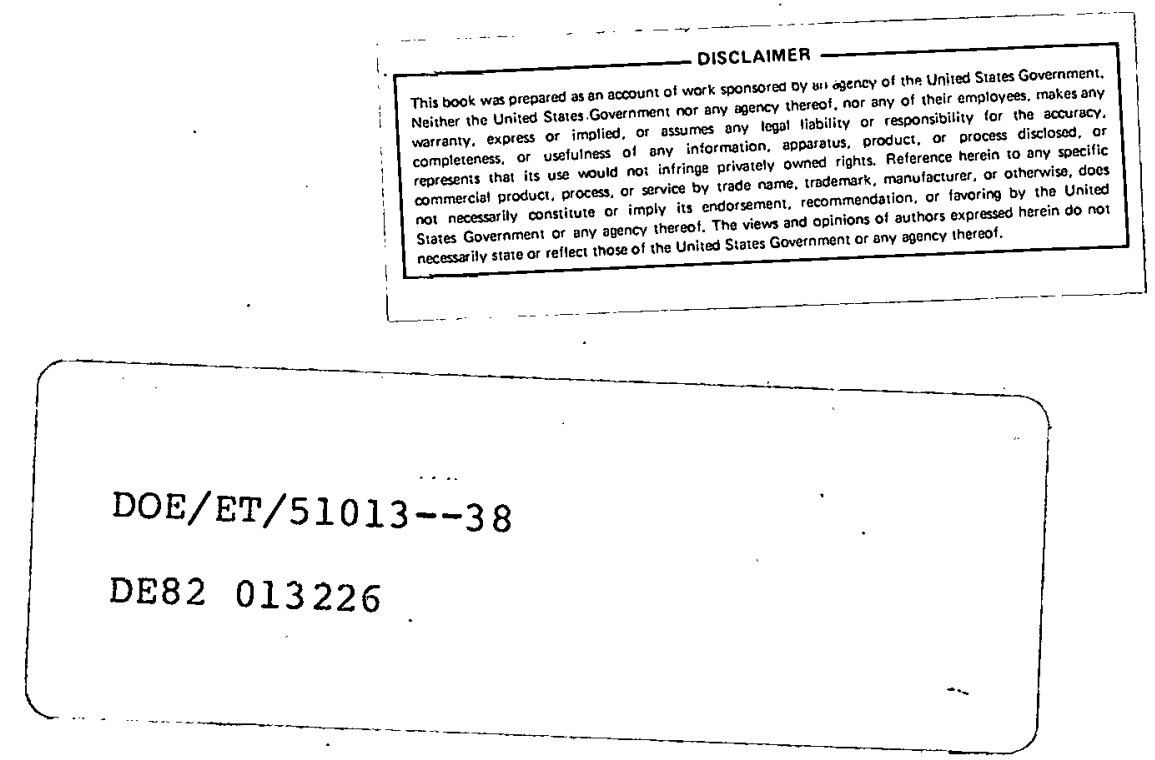

*To appear in Proceedings of Third Joint Varenna-Grenoble International Symposium on Heating in Toroidal Plasmas, Grenoble, France, March 22-27, 1982. 


\title{
ANTENNA-PLASMA COUPLING THEORY FOR IORF HEATING OF LARGE TOKAMAKS
}

\author{
A. Ram and $\Lambda$. Bers \\ Massachusetts Institute of Technology \\ Plasma Fusion Center \\ Cambridge, Massachusetts 02139, U.S.A.
}

A number of experiments (TFR, PLT) have reported significant heating of ions in a plasma by waves in the ion-cyclotron range of frequencies (ICRF). These waves are excited in the plasma by an antenna structure consisting of a current carrying conductor near the plasma wall in the shadow of the limiter. An effective coupling of the external radiofrequency ( $\mathrm{rf}$ ) power to the plasma is achieved when these rf current carrying conductors are shielded from the plasma by a metal screen which locally shorts out the toroidal component of the electric field leaving the poloidal component unaffected. The screen also prevents the plasma from penetrating to the rf conductor. In this paper we study the coupling characteristics of such an antenna structure by analysing a model where a thin current sheet is placed between a fully conducting wall and a sheet of anisotropic conductivity representing the screen. The inhomogeneous plasma in the shadow of the limiter is assumed to extend from the screen onwards away from the antenna. The excitation of the fields inside the plasma are found by analysing the radiation properties of this current sheet antenna. We assume that the current distribution of the antenna is given and that the fields excited inside the plasma are absorbed in a single pass. In all experiments to-date the cross-sectional plasmas are relatively small so that the rf conductor is a half-loop around the plasma in the poloidal direction. However, for reactor size plasmas this cannot be done and the antenna dimensions will be small compared to the plasma cross-sections. We, thus, assume an antenna of finite poloidal and toroidal extent with dimensions sniall compared to the plasma minor radius. We further approximate the coupling geometry by a slab model (Figure 1). The $x$-axis is taken to be along the plasma inhomogeneity, the $y$-axis along the poloidal direction and the $z$-axis along the toroidal magnetic field.

The fields in the vacuum and the plasma are Fourier transformed in $y$ and $z$ and are taken to have a dependence of the form $\exp \left(i k_{y} y+i k_{i} z-i \omega t\right)$. The plasma in the coupling region near the screen is assumed to be described by its cold dielectric tensor:

$$
\left(\begin{array}{ccc}
K_{\perp} & -i K_{X} & 0 \\
i K_{X} & K_{\perp} & 0 \\
0 & 0 & K_{\|}
\end{array}\right)
$$

where 


$$
\begin{aligned}
& K_{\perp}=1-\frac{\omega_{p e}^{2}}{\omega^{2}-\omega_{c e}^{2}}-\sum_{i} \frac{\omega_{p i}^{2}}{\omega^{2}-\omega_{c i}^{2}} \\
& K_{X}=\frac{\omega_{p e}^{2}}{\omega_{c e}^{2}-\omega^{2}} \frac{\omega_{c e}}{\omega}+\sum_{i} \frac{\omega_{p i}^{2}}{\omega^{2}-\omega_{c i}^{2}} \frac{\omega_{c i}}{\omega} \\
& K_{\perp}=1-\frac{\omega_{p e}^{2}}{\omega^{2}}-\sum_{i} \frac{\omega_{p i}^{2}}{\omega^{2}}
\end{aligned}
$$

the summation being over the ion-species, $\omega_{2}$ is the cyclotron frequency, $\omega_{p}$ is the plasma frequency and, $\omega$ is the external frequency. The inhomogeneity in the toroidal magnetic field is introduced through $\omega_{c}$ and that in the density through $\omega_{p}$. The cold plasma dispersion relation leads to the existence of two components of the ICRF wave - the slow and the fast modes. These waves decouple as $\left|K_{\|}\right| \gg\left|K_{\perp}\right|, K_{X}$ inside the plasma. The slow wave has a resonance at the point where $\left|K_{\perp}\right|=0$ which occurs at low densities near the edge of the plasma. In order to avoid the coupling to the slow wave we choose the density at the screen to be such that the slow wave is not excited inside the plasma. This density also ensures that $\left|K_{\|}\right| \gg\left|K_{\perp}\right|, K_{x}$ from the screen onwards into the plasma. So we consider only the coupling to the fast wave. The fast wave $z$-component of the electric field in the plasma $E_{z}^{p}$ is zero. The other two components of the electric field inside the plasma are given by':

$$
\begin{gathered}
E_{x}^{p}=\frac{i}{K_{\perp}-n_{y}^{2}-n_{z}^{2}}\left(n_{y} \frac{c}{\omega} \frac{d E_{y}^{p}}{d x}+K_{X} E_{y}^{p}\right) \\
\frac{d^{2} E_{y}^{p}}{d x^{2}}+f(x) \frac{d E_{y}^{p}}{d x}+g(x) E_{y}^{p}=0
\end{gathered}
$$

where $n_{y, z}=c k_{y, z} / \omega$

$$
\begin{gathered}
f(x)=\frac{-n_{y}^{2}}{\left(n_{z}^{2}-K_{\perp}\right)\left(n_{y}^{2}+n_{z}^{2}-K_{\perp}\right)} \frac{d K_{\perp}}{d x} \\
g(x)=\frac{\omega^{2}}{c^{2}}\left\{K_{\perp}-n_{z}^{2}-n_{z}^{2}-\frac{n_{y}(c / \omega)\left(d K_{X} / d x\right)-K_{X}^{2}}{n_{z}^{2}-K_{\perp}}-n_{y} \frac{K_{X}}{\left(n_{z}^{2}-K_{\perp}\right)\left(n_{y}^{2}+n_{z}^{2}-K_{\perp}\right)} \frac{c}{\omega} \frac{d K_{\perp}}{d x}\right\}
\end{gathered}
$$

Previously, the field solutions for finite $n_{y}$ were examined analytically for the Eqs. (5-8) with all the derivatives of the dielectric tensor elements set to zero ${ }^{2}$. In this paper we give the results of solving (numerically) Eqs. (5-8) without neglecting these derivatives. Only those solutions which give power flow into the plasma are considered.

The fields in the free space region between the wall and screen, and containing the current sheet, are described by a superposition of the full set of transverse-electric (TE) and transverse-magnetic (TM) modes. (The TEM fields related to the feed of the antenna sheet are ignored). These fields are given by: 


$$
\begin{aligned}
& E_{x}^{v}=\frac{\beta^{2}}{\omega \epsilon_{0}}\left(H_{+}^{s} e^{-\gamma x}+H_{-}^{s} e^{\gamma x}\right) \\
& E_{y}^{v}=-\frac{i \gamma}{\omega \epsilon_{0}} k_{y}\left(H_{+}^{s} e^{-\gamma x}-H_{-}^{s} e^{\gamma x}\right)+k_{z}\left(E_{+}^{s} e^{-\gamma x}+E_{-}^{s} e^{\gamma x}\right) \\
& E_{z}^{v}=-\frac{i \gamma}{\omega \epsilon_{0}} k_{z}\left(H_{+}^{s} e^{-\gamma x}-H_{-}^{s} e^{\gamma x}\right)-k_{y}\left(E_{+}^{s} e^{-\gamma x}+E_{-}^{s} e^{\gamma x}\right) \\
& s=I, I I ; \quad \beta^{2}=k_{y}^{2}+k_{z}^{2}, \quad \gamma^{2}=\beta^{2}-\frac{\omega^{2}}{c^{2}}
\end{aligned}
$$

wherc $s=I$ refers to the vacuum region between the wall and the antenna, and $s=I I$ to the region between the antenna and the screen. Setting $H_{+}^{s}=H_{-}^{s}=0$ gives the TE mode and setting $E_{+}^{s}=E_{-}^{s}=0$ gives the TM mode. We assume that the antenna is carrying a current in the $y$ direction at frequency $\omega$. with the current density given by:

$$
\bar{J}=K_{0} \delta(x) F(y) G(z) \hat{y}
$$

where $K_{0}$ is the surface current density amplitude and $F(y)$ and $G(z)$ are the dimensionless profile factors of the current. Letting $E_{z 0}$ be the complex amplitude of the electric field component $E_{y}^{p}$ inside the plasma, the constants $H_{+}^{s}, H_{-}^{s}, E_{+}^{s}, E_{-}^{s}$, and $E_{y 0}$ can be determined by satisfying the boundary conditions at the wall, antenna and the screen.

The radiation impedance of the antenna is obtained by applying the complex Poynting theorem at the surface of the antenna ${ }^{2}$.

$$
\begin{gathered}
Z_{A}=\frac{1}{(2 \pi)^{2}} \frac{2}{|I|^{2}} \int_{-\infty}^{\infty} d k_{y} \int_{-\infty}^{\infty} d k_{z}\left(\frac{1}{2} E_{y} H_{z}^{*}\right)_{x=0}=R_{A}+i X_{A} \\
\text { where } I=K_{0} \int G(z) d z \quad \text { is the current }
\end{gathered}
$$

On matching the boundary conditions at the conducting wall, antenna, and the screen the fields at the antenna can be expressed in terms of the fields inside the plasma. Then, the complex impedance can be expressed as:

$$
\dot{Z}_{A}=\frac{\left|K_{0}\right|^{2}}{(2 \pi)^{2}|I|^{2}} \int_{-\infty}^{\infty} d k_{y} \int_{-\infty}^{\infty} d k_{z}\left|\tilde{F}\left(k_{y}\right) \tilde{G}\left(k_{z}\right)\right|^{2} Z_{k}
$$

where the intrinsic impedance, $Z_{k}$, is given by

$$
Z_{k}=\sqrt{\frac{\mu_{0}}{\epsilon_{0}}} \frac{e^{2 \gamma a}-1}{2\left(e^{2 \gamma(a+b)}-1\right)}\left[\left(e^{2 \gamma b}-1\right) \frac{i \omega}{c \gamma}\left(n_{y}^{2}-1\right)-2 \sqrt{\frac{\epsilon_{0}}{\mu_{0}}}\left(\frac{\tilde{E}_{y}^{p}(x=b)}{K_{0}}\right) e^{\gamma b}\right]
$$

$\tilde{F}\left(k_{y}\right), \tilde{G}\left(k_{z}\right)$ are the Fourier transforms of $F(y), G(z)$ respectively, and $\tilde{E}_{y}^{p}(x)=E_{y}^{p}(x) /\left\{\tilde{F}\left(k_{y}\right) \tilde{G}\left(k_{z}\right)\right\}$ 
'To determine $\tilde{E_{y}^{p}}$ we solve the Eqs. (5-8) by assuming an outgoing wave at a short distance away from the screen inside the plasma. Then the differential equation for $E_{y}^{p}(\mathrm{Eq} .(6))$ is integrated backwards in $x$ to determine $\tilde{E}_{y}^{p}$ at the screen $(x=b)$.

The first term in the expressio 1 of $Z_{k}$ is purely imaginary and contributes only to the reactive part of the impedance. This would be the radiation impedance of the antenna if the screen were to be replaced by a perfectly conducting wall. If we choose the antenna spectrum to be uniform in $y$ and $z$, this reactive part has a logarithmic singularity as $k_{y} \rightarrow \infty$. This singularity is a consequence of the infinite charge required at the antenna ends in $y$ to maintain the assumed uniform current. The experimental situation is closer to that shown in Figure (2) where the feed lines to the antenna are included. Here the current flows smoothly through tl:e antenna and there is no singularity. We approximate this situation by having the antenna in a finite sized rectangular box with fully conducting walls whose $y$ dimension is the same as the length of the antenna in $y$ and whose $z$ dimension is larger than the antenna length in $z$ (Figure 3). Then the singularity at the $y$-ends of the antenna is removed and this should give a good approximation to the reactive loading of the antenna in an actual situation. This particular problem can again be solved in terms of the free-space modes of the box. The reactive part of the antenna impedance is found to be:

$$
\begin{gathered}
X_{A}=\frac{\omega \mu_{0}\left|K_{0}\right|^{2}}{|I|^{2} d L_{y}} \sum_{m, n=0}^{\infty}\left(\frac{4 m^{2} \pi^{2}}{L_{y}^{2} k_{0}^{2}}-l\right) \frac{1}{\bar{a}} \frac{\left(e^{2 \bar{a} b}-1\right)\left(e^{2 \bar{\pi} a}-1\right)}{e^{2 \bar{a}(a+b)}-1}\left|F_{m}\right|^{2}\left|G_{n}\right|^{2} \\
\text { where } \quad \bar{a}^{2}=\frac{4 m^{2} \pi^{2}}{L_{y}^{2}}+\frac{(2 n+1)^{2}}{d^{2}} \frac{\pi^{2}}{4}-k_{0}^{2}, \quad k_{0}=\frac{\omega}{c} \\
F_{m}=\int_{\frac{-L_{y}}{2}}^{\frac{L_{y}}{2}} d y F(y) \cos \left(\frac{2 m \pi y}{L_{y}}\right), \quad G_{n}=\int_{-d}^{d} d z G(z) \cos \left\{\frac{(2 n+1) \pi}{2 d} z\right\}
\end{gathered}
$$

and, $2 d\left(>L_{z}\right)$ is the length of the box in the $z$-direction. If we now assume a uniform current profile, then $F_{m}$ is nonzero for $m=0$ only. Thus, the double sum in Eq. (16) is reduced to just a single sum over $n$ which converges rapidly to give a finite contribution to the reactive impedance. The contribution to the total reactive impedance from the second term of Eq. (15) is found to be very small compared to the reactive impedance evaluated from Eq. (16). The resistive part of the antenna impedance is just the real part of the second term of Eq. (15).

The power flow into the plasma is given by

$$
P_{P L}=\operatorname{Re}\left[\frac{1}{2} \frac{1}{(2 \pi)^{2}} \int_{-\infty}^{\infty} d k_{y} \int_{-\infty}^{\infty} d k_{z} E_{y}^{p} H_{z}^{p^{*}}\right]
$$

As an example, we apply the above results to a tokamak plasma consisting of just deuterium and electrons with the following parameters: major radius $=3 \mathrm{~m}$., minor radius (to the screen) $=1.27 \mathrm{~m}$., density at the screen $=2 \times 10^{11} \mathrm{~cm}^{-3}$, peak density (at center) $=$ $5 \times 10^{13} \mathrm{~cm}^{-3}$, toroidal magnetic field $=35 \mathrm{kG}, \omega=2 \omega_{C D}=3.35 \times 10^{8} \mathrm{sec}^{-1}, a=5 \mathrm{~cm}$, $b=3 \mathrm{~cm}, L_{y}=60 \mathrm{~cm}, L_{z}=40 \mathrm{~cm}, d=25 \mathrm{~cm}$ and a uniform current profile in $y$ and $z$. With the above choice of edge density we make sure that the slow wave is not excited at the 
edge. For a parabolic density profile the real part of the integrand of Eq. (14) is plotted as a function of $n_{y}$ and $n_{z}$ in Figure (4). We find that the major contribution to the resistive imipedance of the antenna comes from the region $n_{y} \neq 0$. The total resistive impedance is calculated to be $R_{A}=9.58 \Omega$. If we set $n_{y}=0$ in Eqs. (5-8) then we would get a resistive impedance of $6.7 \Omega /$ meter of antenna length in $y$. For an antenna with $L_{y}=60 \mathrm{~cm}$, this would give a resistive impedance of $4 \Omega$. This is less than half the value obtained when we take into account the appropriate $n_{y}$ spectrum. In our previous work ${ }^{2}$ where we accounted for the $n_{y}$ spectrum, but the derivatives of the dielectric tensor elements were set to zero, a total resistive impedance of $21.0 \Omega$ was obtained for the same parameters and profiles; this is more than twice the value obtained from the exact theory which does not set the derivatives of the dielectric tensor elements to zero.

From our results we find that the major contribution to $R_{A}$ comes from the $n_{y}<0$ part of the spectrum. We, thus, conclude that a set of poloidal antennae phased to excite a current spectrum biased towards $n_{y}<0$ would couple more effectively to the p!asma. For the above parameters, we also find that $\left(2 P_{P L} / I^{2}\right)$ of Eq. (17) is $9.4 \Omega$. Comparing this to $R_{A}=9.58 \Omega$ we find that almost all of the power emitted from the antenna is going into the plasma. The reactive impedance from Eq. (16) is calculated to be $X_{A}=22.5 \Omega$. This reactive impedance is completely inductive.

If we keep all parameters fixed as above but change the density profile to a gaussian, we get $R_{A}=18.3 \Omega$. Hence, for the same edge density a more gradual increase in the density from the edge leads to better coupling.

This work is supported by DOE Contract No. DE-AC02-78ET-51013.

[1] : A. Bers, J. Jacquinot, G. Lister, 2nd Joint Grenoble- Varennes Int. Symp., Como, Italy, Sept: 1980 and Report EUR-CEA-FC-1066 Fontenay-aux-Roses, France, Sept. 1980.

[2] A. Bers, L. Harten, A. Ram, Proceedings of the Fourth Topical Conference on RF Plasma Heating, 1981, Austin, Texas, pp. A16-1-A16-4. 


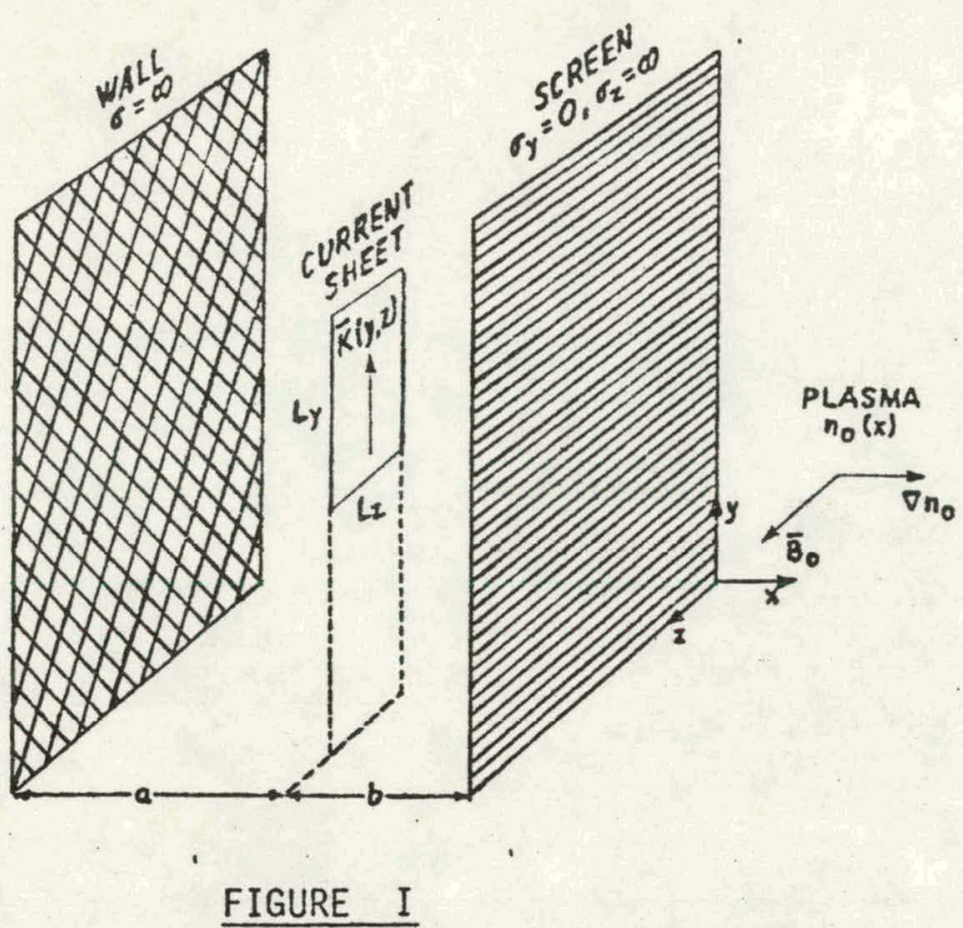

Slab geometry model used for the analyses.

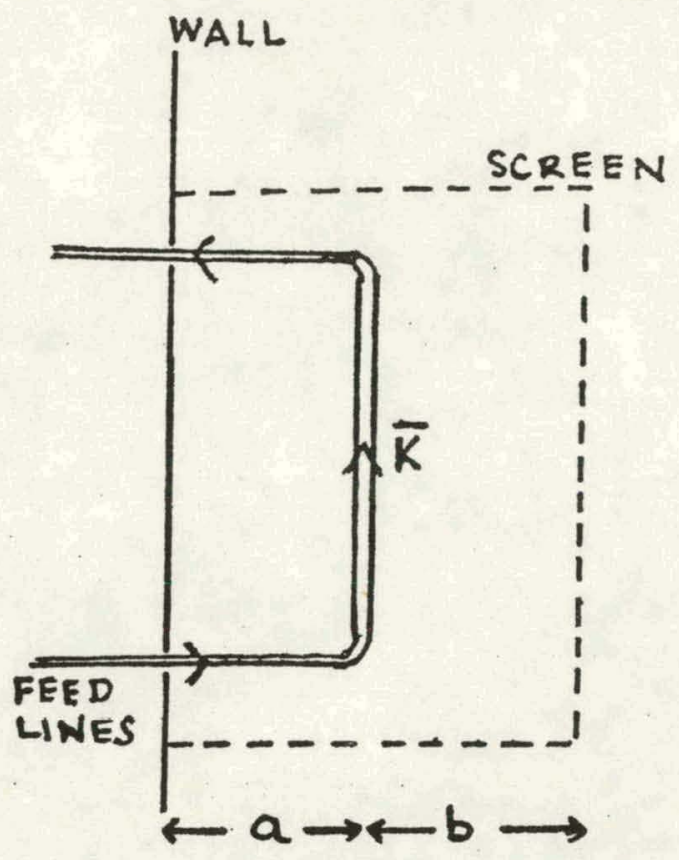

FIGURE 2

Schematic representation of the actual coupling structure.

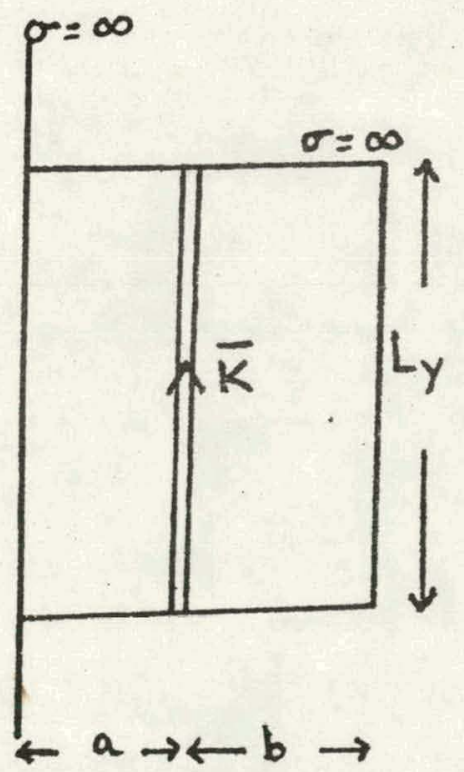

FIGURE 3

Geometry used for evaluating the reactive impedance of the antenna. The dimension in $z$ is $2 d$.

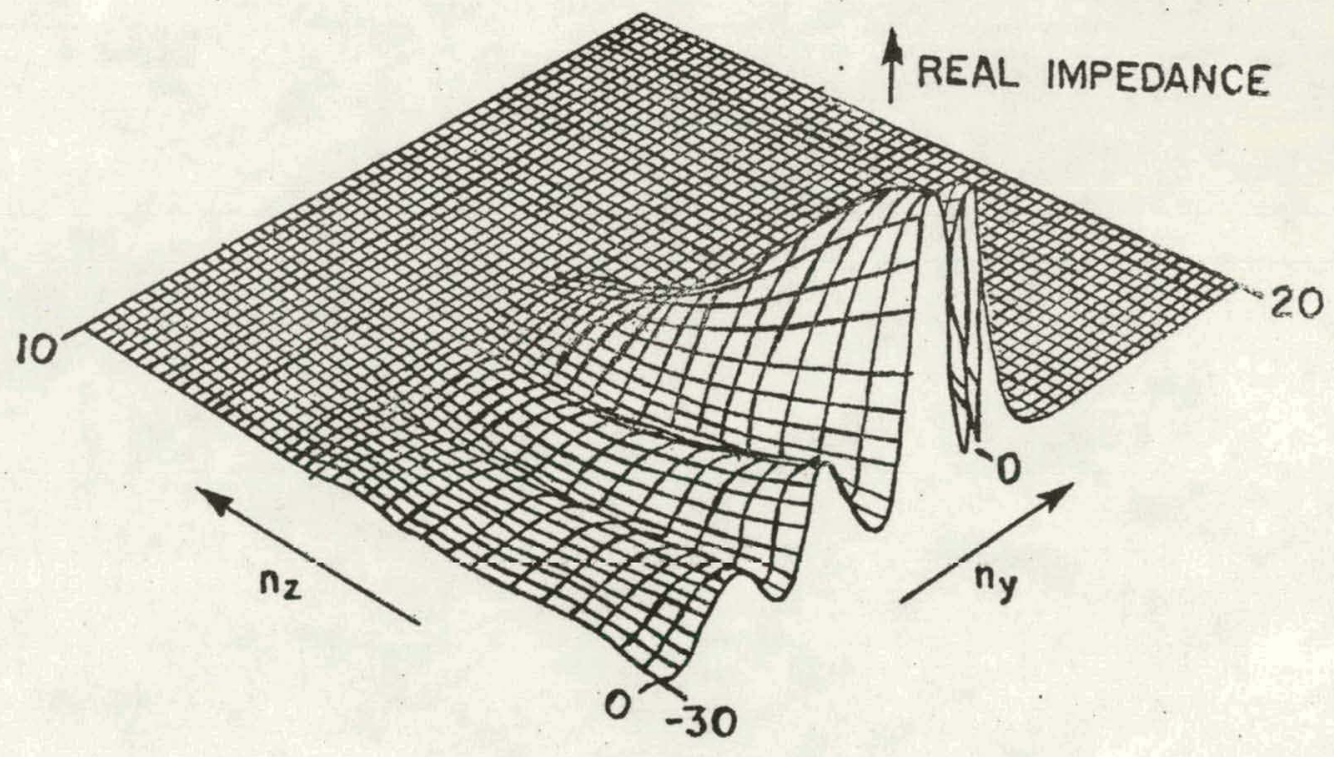

FIGURE 4

Real part of the antenna impedance plotted as a function of $n_{y}$ and $n_{z}$. 
G. Bekefi

36-213

A. Bers

38-260

D. Cohn

NW16-250

B. Coppi

26-201

R.C. Davidson

NWר6-202

T. Dupree

38-172

S. Foner

NW14-3117

J. Freidberg

38-160

A. Gondhalekar

NW7 6-278

M. 0. Hoenig

NW16-176

M. Kazimi

NW172-209

L. Lidsky

38-174

E. Marmar

NW76-280

J. Mccune

$31-265$

J. Meyer

24-208

D.B. Montgomery

NWา $6-140$

J. Moses

ME-43-514

J. Pappas

:11176-272
R.R. Parker

NWI6-288

N.T. Pierce

NW16-186

P. Politzer

NW16-286

M. Porkolab

36-293

H. Praddaude

NW74-3101

D. Rose

24-210

J.C. Rose

NW16-189

R.M. Rose

4-132

B.B. Schwartz

NW14-5121

R.F. Post

NWL2T-203

L.D. Smullin

38-294

R. Temkin

NW16-254

N. Todreas

NW13-202

J.E.C. Will 1 iams

NW14-3210

P. Wolff

36-419

T. F. Yang

NW16-164

Industrial Liaison Office

ATTN: Susan Shansky

iHonthly List of Publications

$39-513$
MIT Libraries

Collection Development

ATTN: MIT Reports

I4E-210

B. Colby

PFC Library

NW16-255 
National

Argonne National Laboratory

Argonne, IL 60439

ATTN: Library Services Dept.

Battelle-Pacific Northwest Laboratory P.O. Box 99

Richiand, WA 99352

ATTN: Technical Information Center

Brookhaven National Laboratory

Upton, NY . 11973

ATTN: Research Library

U.S. Dept. of Energy

Washington, D.C. 20545

ATTN: D.O.E. Library

Roger Derby

Oak Ridge National Lab.

ETF Design Center

Bldg. 9204-1

Oak Ridge, TN 37830

General Atomic Co.

P.0. Box 81608

San Diego, CA 92138

ATTN: Library

Lawrence Berkeley Laboratory

1 Cyclotron Rd.

Berkeley, CA 94720

ATTN: Library

Lawrence Livermore Laboratory

UCLA

P.O. Box 808

Livermore, CA 94550

Oak Ridge National Laboratory

Fusion Energy Div. Library

Bldg. $9201-2, \mathrm{~ms} / 5$

P.O. Box "Y"

Oak Ridge, TN 37830
Dr. D. Overskei

General Atomic Co.

P.0. Box 81608

San Diego, CA 92138

Princeton Plasma Physics Laboratory Princeton University P.0. Box 451

Princeton, NJ 08540

ATTN: Library

Plasma Dynamics Laboratory Jonsson Engineering Center

Rensselaer Polytechnic Institute Troy, NY 12181

ATTN: Ms. R. Reep

University of Wisconsin

Nuclear Engineering Dept.

1500 Johnson Drive

Madison, WI 53706

ATTN: UV Fusion Library 


\section{International}

Professor M.H. Brennan

Willis Plasma Physics Dept.

School of Physics

University of Sydney

N.S.W. 2006, Australia

Division of Plasma Physics

Institute of Theoretical Physics

University of Innsbruck

A-6020 Innsbruck

Austria

c/o Physics Section

International Atomic Energy Agency Wagramerstrasse 5

P.0. Box 100

A-1400 Vienna, Austria

Laboratoire de Physique des Plasmas

c/o H.W.H. Van Ande?

Dept. de Physique

Universite de Montreal

C.P. 6128

Montreal, Que H3C 3J7

Canada

Plasma Physics Laboratory

Dept. of Physics

University of Saskatchewan

Saskatoon, Sask., Canada S7N OWO

The Library

Institute of Physics

Chinese Academy of Sciences

Beijing, China

Mrs. A. Wolff-Degives

Kernforschungsaniage Julich $\mathrm{GmbH}$

Zentralbibliothek - Exchange Section

[-5170 Julich - Postfach 1913

Federal Republic of Germany

Preprint Library

Central Research Institute for Physics

H-1525 Budapest, P.0. Box 49

Hungary

Plasma Physics Dept.

Israel Atomic Energy Commission

Soreq Nuclear Research Center

Yavne 70600

Israe]
The Librarian (Miss DePalo)

Associazione EURATOM - CNEN Fusione C.P. 65-00044 Frascati (Rome)

Italy

Librarian

Research Information Center

Institute of Plasma Physics

Nagoya University

Nagoya, 464

Japan

Dr. A.J. Hazen

South African Atomic Energy Board

Private Bag X256

Pretoria 0001

South Africa 


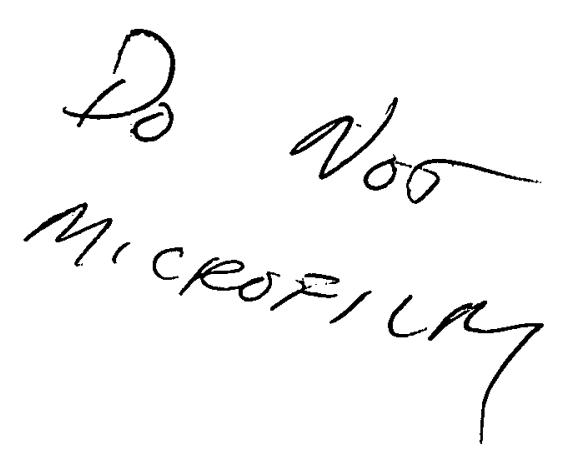

